\title{
Der Zusammenhang der Atemkräfte und ihre Abhängigkeit vom Dehnungszustand der Atmungsorgane.
}

\author{
Von \\ Fritz Rohrer, \\ Assistenzarzt der mediz. Poliklinik Tübingen.
}

(Mit 4 Textfiguren.)

Inhaltsverzeichnis.

Einleitung. . . . . . . . . . . . . . . . . 419

I. Die an der Oberflächeneinheit der Brusthöhle wirkenden Kräfte . . . 421

A. Zusammenhang und Ausgleich der Atemkräfte . . . . . . . 421

1. Die Spannung des Brusthöhleninhaltes und der innere Spannungsausgleich. . . . . . . . . . . . . . . . 421

2. Die Spannung in der Umgebung der Brusthöhle und der äussere Spannungsausgleich . . . . . . . . . . . 422

B. Die Verhältnisse bei Muskelerschlaffung . . . . . . . . . . . . 424

1. Messung der elastischen Spannungsresultante bei Muskelerschlaffung . . . . . . . . . . . . . 424

2. Die elastische Spannung der Brusthöhlenumgebung . . . . 428

C. Die Verhältnisse bei maximaler Muskelanstrengung . . . . . . . 431

1. Die maximale inspiratorische und exspiratorische Kraft. . . . 431

2. Die maximale inspiratorische und exspiratorische Muskelkraft. 453

D. Indirekte Messung der elastischen Kräfte und die Verhältnisse beim Emphysem. . . . . . . . . . . . . 485

II. Die an der Gesamtoberfläche der Brusthöhle wirkenden Krälte. . . . 438

Zusammenfassung ................. 442

Die äussere Atmung ist ein rhythmischer Bewegungsvorgang, an welchem ein grosser Teil des Körperstammes und seiner Inhalte sich beteiligt. Die einzelnen Organe dieses morphologisch überaus komplizierten Systems erfahren Lage- und Formänderungen, die so koordiniert sind, dass der obere Abschnitt der Leibeshöhle, der Brustraum und sein Inhalt, dabei abwechselnd Volumvergrösserung und Verminderung erfährt. 
Wenn man die Lehr- und Handbücher der Physiologie und Spezialarbeiten über die Atmungsmechanik durchsieht, gewinnt man den Eindruck, dass bei der Untersuchung dieses Vorganges bisher das Hauptgewicht auf die Feststellung der kinematischen Verbältnisse, der Koordination einzelner Lage-, Form- und Volumänderungen, der Betätigung bestimmter Muskelgruppen und ihre Bedingtheit dureh Form und Anordnung des Skelettes und der Weichteile, gelegt wurde. Diese Tendenz kommt auch zum Ausdruck in der grossen Zahl von Apparaten, die $z u$ diesem Zweck konstruiert wurden ${ }^{1}$ ). Die röntgenologischen Untersuchungen bewegen sich ebenfalls in dieser Richtung. Über die Grösse der bei der Atmung wirkenden muskulären, elastischen usw. Kräfte sind im einzelnen spärliche Angaben vorhanden, dagegen sind die Vorstellungen uber ihr Zusammenarbeiten wenig entwickelt ${ }^{2}$ ). Es scheint mir dies ein Mangel, indem, wie die Geschichte der Physik mehrfach zeigt, bei komplizierten Bewegungsvorgängen, durch die Kenntnis der veranlassenden Kräfte und ihres Ineinandergreifens die Einsicht in den Ablauf des Vorganges an Tiefe und oft auch die Darstellung an Einfachheit gewinnt.

Die nachstehenden Ausführungen stellen einen Versuch dar für die verschieltenen Dehnungszustände der Atemorgane, die hier zunächst als statische Zustände angenommen sind, die Grösse und gegenseitige Abhängigkeit der Atemkräfte festzustellen. Die Dynamik der Atmung wird Gegenstand einer späteren Untersuchung sein.

Die Messung der statischen Atemkräfte geschieht durch Bestimmung dè Druckerhöhung bzw. Erniedrigung, welche sie der Thoraxluft zu erteilen vermögen. Diese Druckdifferenz, welcher die Atemkräfte das Gleichgewicht halten, wirkt ringsum an der Brusthöhlenoberfläche. IDie Messung der Druckdifferenz mit einem Manometer orientiert zunächst nur über die Kräfte an der Oberflächeneinheit der Brusthöhle. Um die Gesamtkräfte zu erhalten, sind diese Werte mit der Oberflächengrösse des Brustraumes zu multiplizieren.

1) Tigerstedt, Handb. d. physiol. Methodik Bd. 2 Abt. 2 S. 4-18.

2) Zum Beispiel: Boruttan in Nagel's Handb. d. Physiol. Bd. 1 S. 21-25. - R. du Bois-Reymond, Ergebn. d. Physiol. v. Asher u. Spiro Bd. 1 (2) S. 377-402. 1902. - Minkowski, Handb. d. allgem. Pathol. v; Krehl u. Marchand Bd. 2. - Waldenburg, Pneumatische Behandlung S. 69-81. 1880. - Tendeloo, Studien über die Ursachen der Lungenkrankheiten. Physiol. Teil S. 1-33. 1902. 


\section{Die an der Oberflächeneinheit der Brusthöhle wirkenden Kräfte.}

\section{A. Zusammenhang und Ausgleich der Atemkräfte.}

\section{Die Spannung des Brusthöhleninhaltes und der innere Spannungsausgleich.}

Für die an der Oberflächeneinbeit der Lungen wirkende Kraft fanden wir in einer fruheren Arbeit ${ }^{1}$ ) den Ausdruck $p=p_{\epsilon l}+p_{a l v *}$. $p_{e l}$ ist die bei einem gegebenen Dehnungszustand vorliegende elastische Retraktionskraft pro Quadratzentimeter Oberfläche, $p_{a l v}$ die Druckdifferenz zwischen Lungenluft und Aussenluft; sie ist für Inspiration mit positivem, für Exspiration mit negativem Vorzeichen in die Formel zu setzen.

Wenn $p=0$ wird, ist $p_{\epsilon l}=-p_{u l v}$, d. h. die elastische Re traktionskraft der Lungen ist durch die pneumatische Druckdifferenz messbar, welche ihr das Gleichgewicht hält (Donders). Die elastische Retraktionskraft der Lungen pro Quadratzentimeter Oberfläche ist nach den Untersuchungen $\mathrm{Cl}$ loet $\mathrm{t}$ a's eine lineare Funktion des Lungenvolumens ${ }^{2}$ ). Wir fanden den Ausdruck ${ }^{3}$ ) $p_{\epsilon l}=p_{\epsilon l}+k \cdot Q$ ( $Q$ in Litern, $k=4,5, p_{e l}$ in Zentimeter $\mathrm{H}_{2} \mathrm{O}$ ).

In gleicher Weise wie die Lungen befinden sich auch die Organe des Mediastinums zwischen Sternum, Wirbelsäule, Diaphragma und den beiderseits anliegenden Lungen in Spannung:

Infolge der leichten Verschieblichkeit der Lungen, welche den Hauptvolumanteil des Thoraxraumes füllen, ist ein nahezu jdealer Spannungsausgleich möglich ${ }^{4}$ ), so dass an jeder Flächeneinheit der Brusthöhlenwandung die gleiche, senkrecht nach innen gerichtete Kraftresultante angreift. Die Dehnungsgleichung der Lungen $p=p_{e l_{0}}+4,5 Q+p_{a v}$ gilt daher gleichzeitig auch für den gesamten Brusthöhleninhalt.

Eine Sonderstellung ist bei extremer Inspiration oder Exspiration nur für die medial oberhalb des Hilus gelegenen Lungenabschnitte anzunehmen ${ }^{5}$ ).
1) Pflüger's Arch, Bd. 162 S. 261.
2) P.flüg er's Arch. Bd. 152 S. 339-364.
3) Pflüger's Arch. Bd. 162 S. $28 \dot{2}-283$.
4) Pflüger's Árch. Bd. 162 S. 289.
5) Pflüg'er's Arch. 'Bd. 162 S. 290. 
Eine Sonderstellung besizen ferner infolge der Herztätigkeit die Bluträume des Mediastinums.

Die Spannung dieser Inhalte des Brustraumes überträgt sich nach allen Richtungen auf die Wandungen desselben, die nach unten hin durch das Diaphragma, nach den Seiten durch Sternum, Rippen, Interkostalmuskulatur, Wirbelsäule, nach oben hin durch die Muskulatur und übrigen Organe der oberen Brustapertur gebildet werden, und setzt sich hier ins Gleichgewicht mit den von aussen angreifenden dehnenden Kräften.

\section{Die Spannungen in der Umgebung der Brusthöhle und der äussere Spannungsausgleich.}

Die debnende Kraft $p$, welche in einem bestimmten Zeitpurikt an der Brusthöhlenoberfläche angreift, ist die Resultante aus zwei Kräften.

a) Die eine Komponente sind die muskulären Kräfte, welche teils direkt (Diaphragma), teils auf dem Umweg über das komplizierte Hebelsystem des Thoraxskelettes, oder durch Vermittlung des Bauchhöhleninhaltes (Bauchwandmuskulatur) auf die Brusthöhlenoberfläche einwirken. Der Übertragung der indirekt wirkenden Muskelkräfte geht in jedem Zeitpunkt eine entsprechende elastische Spannung der die Kraft übertragenden Skelettabschnitte und Weichteile parallel. Diese den momentan wirkenden Muskelkräften entsprechenden Spannungen können wir als primäre oder Kraftübertragungs-Spannungen bezeichnen.

b) Als zweite Komponente wirken sekundäre, während einer anderen Atemphase durch Speicherung muskulärer Arbeit erzeugte Spannungsmomente: elastische Spannungen (Rippenknorpel, Bandapparate, Weichteile) und Schweremomente (Gewicht der Thoraxwand, Bauchinhalt, vor allem Lebergewicht). Diese sekundären Spannungsmomente kommen zum Ausdruck in der momentanen Form (Torsion der Rippenknorpel) und Lage der einzelnen Teile der Brusthöhlenumgebung, sie sind abhängig vom Dehnungszustand der Brusthöhle, dagegen unabhängig von der in diesem Zeitpunkt wirkenden Muskelkraft. Im Gegensatz dazu können bei einem bestimmten Dehnungszustand in verschiedenstem Maasse und auch in verschiedenem Sinn (inspiratorisch oder exspiratorisch) Muskelkräfte wirken und entprechende Kraftübertragungs-Spannungen vorhanden sein. Es geht daraus hervor, dass die Kraftübertragung nur geringe 
Deformation der Skelett- und Weichteile erfordert, dass diese oft hohen Spannungen transitorisch sind und bei einem gegebenen Dehnungszustand nicht als gespeicherte Energie nach Aufhören der Muskeltätigkeit zu einer passiven Atembewegung Verwendung finden können, wie die sekundären Spannungen.

Die senkrecht zur Brusthöhlenoberfäche gerichtete Komponente dieser letzteren Spannungsmomente an einer bestimmten Oberflächeneinheit der Brusthöhle sei $p_{\varepsilon l \text { thor }}$, die dorthin fortgeleitete senkrecht wirkende muskuläre Kraft $p_{m u s k}$ Es ist dann die dehnende Kraft an dieser Stelle:

$$
p=p_{\text {musk }}+p_{\text {el thor. }}
$$

Da diese Kraft gleich und entgegengesetzt dem Zug des Brustinhaltes ist, gilt die Beziehung:

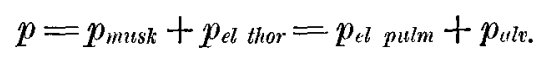

An jeder Oberflächeneinheit des Brustraumes ist in einem bestimmten Zeitpunkt die innere Spannungsresultante. gleich gross wie die äussere. Weil durch intrathorakalen Druckausgleich die erstere überall von gleicher Grösse ist, gilt dasselbe für die äussere Spannungsresultante.

- Dieser Ausgleich der äusseren Spannungen ist die Folge und geht auf dem Wege des inneren Spannungsausgleiches im Brustraum. Zwischen den Brustwandungsabschnitten befindet sich der gleichmässig gespannte Brustinhalt, welcher wie eine ideale Flüssigkeit alle an der Wandung ansetzenden Zug- oder Druckkräfte unter sich zum Ausgleich bringt.

Normalerweise sind die elastischen und muskulären Kraftentwicklungsmöglichkciten derart gleichmässig in der Brusthöhlenumgebung verteilt, dass alle Wandungsabschnitte, indem sie teils in Ruhe bleiben und nur durch zunehmende elastische und muskuläre Spannung den Zug des Brustinhaltes tragen (Wirbelsäule, hintere Thoraxfläche, Tonus des Arrector trunci) oder indem sie im Sinne der gerade vorhandenen Respirationsphase sich bewegen (seitliche und vordere Thoraxfläche, Diaphragma), der Bcdingung des Spannungsausgleichs genügen.

Wenn unter pathologischen Verhältnissen die gleichmässige Verteilung der Kraftentwicklungsmöglichkeiten in der Brusthöhlenumgebung gestört ist, können normalerweise respiratorisch ausgiebig bewegte Brustwandabschnitte, infolge Spannungsausgleiches mit den übrigen Abschnitten, viel weniger bewegt werden, oder sich in umgekehrtem Sinn wie diese bewegen und so die Aufgabe der Atembewegung, die Volumänderung des Brustraumes beeinträchtigen, z. B. inspiratorisches Emporsteigen des Diaphragmas bei Phrenicuslähmung (ähnlich auch die inspiratorische Ansaugung des Mediastinums bei halbseitigem offenem Pneumothorax). Auch hier ist in jedem Zeitpunkt die äussere 
Spannung an allen Brustwandabschnitten gleich, zum Beispiel das gelähmte Zwerchfell wird dem durch den Brustinhalt übertragenen Zug der übrigen Brusthöhlenwand folgend, inspiratorisch so weit emporgesogen, bis seine elastische Spannung dem Zuge Gleichgewicht hält.

\section{B. Die Verhältnisse bei Muskelerschlaffung.}

\section{Messung der elastischen Spannungsresultante bei Muskel-} erschlaffung.

Die Kraft, welche die Muskulatur auf die Oberflächeneinheit ausübt, ist nach obenstehender Gleichung:

$$
p_{\text {musk }}=-p_{\text {el thor }}+p_{e l p u l m}+p_{a b v}=\Sigma p_{e l}+p_{a l v} \text {. }
$$

Die Grösse der äusseren sekundären Spannungen, $p_{c l}$ thor , kann nicht direkt bestimmt werden, dagegen gibt uns die letztere Gleichung den Weg zur Bestimmung von $\Sigma p_{e l}$ für die verschiedenen Dehnungszustände der Atemorgane.

Wenn man bei irgend einemDehnungszustand die Luftwege abschliesst und die Atemmuskulatur erschlaffen lässt, wird $p_{\text {musk }}=0$ und $\Sigma_{p_{e l}}=-p_{a l v}$. Die sekundäre Spannkraft des Brusthöhleninhaltes und ihrer Umgebung ist pro Oberflächeneinheit für irgendeinen Dehnungszustand der Atemorgane, gleich der intrathorakalen Druckdifferenz bei Muskelerschlaffung.

Für eine 28 Jahre alte, gesunde männliche Versuchsperson von $166 \mathrm{~cm}$ Körperlänge und 3,3 Liter vitaler Kapazität, ergaben sich in sitzender Stellung, bei Muskelerschlaffung und Abschluss der Luftwege durch ein offenes Wassermanometer für je 1/2 Liter auseinanderliegende Dehnungszustände, die einzeln durch Exspiration in ein Trockenspirometer von Barnes, ausgehend von maximaler Inspirationslage erhalten wurden, folgende Werte für $\Sigma_{p_{c l}}$ :

\begin{tabular}{l|c|c|c|c|c|c|c|c}
\hline & \multicolumn{7}{|c}{ Exspiration in Litern } \\
\cline { 2 - 8 } & 3,3 & $\mathbf{3}$ & 2,5 & $\mathbf{2}$ & 1,5 & 1 & 0,5 & 0 \\
\hline$\Sigma p_{e l}$ : Zentimeter $\mathrm{H}_{2} \mathrm{O}$ & -36 & -26 & -10 & -2 & 4 & 10 & 18 & 28
\end{tabular}

Die Messungen wurden ohne nochmalige Kenntnisnahme der früheren Werte später nachkontrolliert. Es ergaben sich für die vier mittleren Werte Schwankungen bis $\pm 1 \mathrm{~cm} \mathrm{H}_{2} \mathrm{O}$, für die äusseren bis $\pm 2 \mathrm{ccm} \mathrm{H}_{2} \mathrm{O}$.

In Fig. 1 sind über den Lungenvolumina als Abszissen diese Druckwerte als Ordinaten aufgetragen. Die Kurve $(I)$ des intrathorakalen Druckes bei Muskelerschlaffung schneidet die Abszisse bei. 
der elastischeri Gleichgewichtslage der Atmungsorgane: gewöhnlicher Exspirationszustand. Oberhalb dieses Dehnungszustandes bedingt $\mathbf{\Sigma} p_{\epsilon l}$ einen Überdruck, unterbalb einen Unterdruck im Thorax.. Die Kurve des Druckverlaufs ist in dem für die Atmung hauptsächlich in Betracht fallenden Dehnungsbereich von ca. $1 / 2$ Liter unterhalh lis ca. $1^{1 / 2}$ Liter oberhalb der Gleicbgewichtslage von ancähernd gleichmässiger Neigung, während sie gegen die extreme Inspiration und noch mehr gegen die extreme Exspirpation hin einen zunehmend steileren Verlauf nimmt.

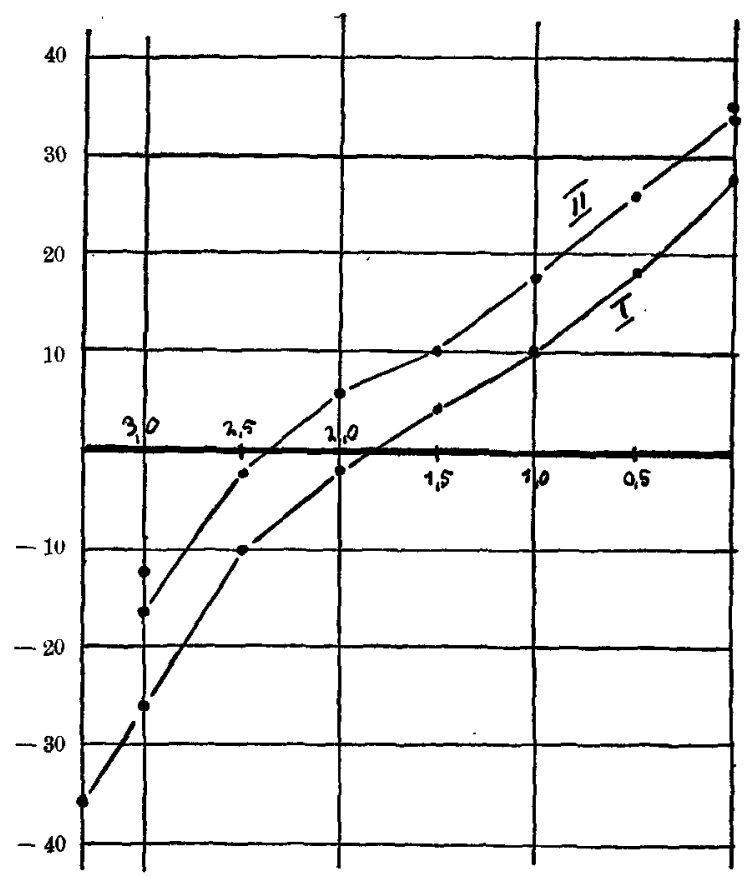

Fig. 1. Abszissenwerte nach links $=$ Exspiration in Litern von maximaler Inspiration aus. Ordinatenwerte $=$ Druck in Zentimetern $\mathrm{H}_{2} \mathrm{O}$ bei al)geschlossener Glottis und Muskelerschlaffung. - I Kurve der elastischen Spannungsresultante im Sitzen. II Kurve der elastischen Spannungsresultante im Liegen.

In dem mittleren 2 Liter umfassenden Dehnungsbereich, zwischen 0,5 und 2,5 der Tabelle, wo $\Sigma p_{e l}$ eine lineare Funktion des Dehnungszustandes ist, findet sich eine Druckänderung von +18 zu $-10=$ $28 \mathrm{~cm} \mathrm{H}_{2} \mathrm{O}$, pro Liter Dehnungsänderung also $14 \mathrm{~cm} \mathrm{H}_{2} \mathrm{O}$ Druckänderung. Wenn in diesem Dehnungsbereich von einem Zustanıl $\Sigma p_{\theta l_{0}}$ aus eine Volumänderung von $Q$-Liter erfolgt, ist der am Schlusse erreichte Spannungswert $\Sigma p_{e l}=\Sigma p_{e l_{0}}+k_{e l}: Q \cdot\left(k_{e l}=14\right)$. 
Wenn der Atemzug von Gleichgewichtslage aus erfolgt ist $\Sigma p_{e l_{0}}=0$ und $\Sigma p_{e l}=14 \cdot Q \mathrm{~cm} \mathrm{H}_{2} \mathrm{O}(Q$ ist inspiratorisch mit positivem, exspiratorisch mit negativem Vorzeichen in die Formel zu setzen.

Da ein Anteil von $p_{e l}$ thor durch Schweremomente geleistet wird, ist es von Interesse, ob die $\Sigma p_{\ell l}$ Kurve bei wechselnder Körperhaltung oder -lage ändert. Messung in aufrechter Körperlage bei gesenkten und bei senkrecht erbobenen Armen ergab keinen Unterschied. Dagegen zeigt sich eine deutliche Erhöhung aller Druckwerte im Liegen und noch mehr im senkrechten Hangen mit dem Kopf nach unten; für letztere Stellung wurden nur die Werte für extreme Inspiration und Exspiration gemessen. Die vitale Kapazität im Liegen bestimmte sich zu 3,0 Liter, im Hangen wurde sie nicht gemessen; wir nehmen den letzteren-Wert als geltend an. Die Werte für aufrechte Körperlage wurden zum Vergleich in folgender Tabelle nochmals beigesetzt.

\begin{tabular}{|c|c|c|c|c|c|c|c|c|}
\hline & \multicolumn{8}{|c|}{ Exspiration in Litern } \\
\hline & 8,3 & 3 & 2,5 & 2 & 1,5 & 1 & 0,5 & 0 \\
\hline Aufrechte Lage: $\Sigma p_{e l} \mathrm{~cm} \mathrm{H}_{2} \mathrm{O}$. & -36 & -26 & -10 & -2 & 4 & 10 & 18 & 28 \\
\hline Liegen: $\Sigma p_{\epsilon l} \mathrm{~cm} \mathrm{H} \mathrm{H}_{2} \mathrm{O} \ldots$ & - & -16 & -2 & 6 & 10 & 18 & 26 & 34 \\
\hline Hangen: $\Sigma p_{e l} \mathrm{~cm} \mathrm{H}_{2} \mathrm{O} \ldots$ & - & -12 & - & - & - & - & - & 35 \\
\hline
\end{tabular}

Wie die Tabelle und Fig. 1 zeigt, ist für die $\Sigma_{p_{\ell l}}$.Kurve im Liegen $(I I)$ der Verlauf höher, aber annähernd parallel dem bei aufrechter Stellung. Zwischen 0,5 und 2,5 ist die Druckänderung pro Liter auch hier $14 \mathrm{~cm}_{2} \mathrm{H}_{2} \mathrm{O}$. Der Schnittpunkt init der $\mathrm{Ab}$ szissenachse, d. h. die elastische Gleichgewichtslage ist näher an die Fxspirationslage herangerückt. Es entspricht dieses Ergebnis der röntgenologischen Beobachtung, dass in Liegen das Zwerchfell höher steht $\left.{ }^{1}\right)$.

In ähnlicher Weise ist die $\Sigma p_{t i}$-Kurve abhängig von Änderungen der elastischen Momente. Schon beim Normalen ist eine solche Änderung möglich durch Anbringung äusserer exspiratorisch wirkender, inspiratorisch sich spannender Vorrichtungen. Es wurde bei der Versuchsperson in der Höhe des Proz. xiphoideus eine elastische Stauungsbinde mit mässiger Spannung angelegt und nun im Sitzen

1) Groede1, Atlas und Grundriss der Röntgendiagnostik in der inneren Medizin S. 46. 1909. 
die gleichen Messungen wie oben wiederholt. Die vitale Kapazität war 3,0-3,1 Liter. Die Werte ohne Binde sind zum Vergleich beigesetzt.

\begin{tabular}{l|r|r|r|r|r|r|r|r|r}
\hline & \multicolumn{7}{|c}{ Exspiration in Litern } \\
\hline & 3,3 & 3 & 2,5 & 2 & 1,5 & 1 & 0,5 & 0 \\
\hline Ulit elast. Binde: $\Sigma p_{c l}\left(\mathrm{~m} \mathrm{H}_{2} \mathrm{O}\right.$ & - & -10 & -2 & 6 & 12 & 20 & 28 & 38 \\
Ohne $", \quad \Sigma p_{\ell l} \mathrm{~cm} \mathrm{H}_{2} \mathrm{O}$ & -36 & -26 & -10 & -2 & 4 & 10 & 18 & 28
\end{tabular}

Die Kurve entspricht etwa derjenigen im Liegen. Interessant ist, dass durch diese Zufügung eines nicht unbeträchtlichen Spannungsmomentes am Thorax (die Gleichgewichtslage wird um $1 / 2$ Liter in exspiratorischer Richtung verschoben) die Neigung der Kurve in den mittleren Partien kaum beeinflusst wird. Zwischen 0,5 und 2,5 ist die Differenz $30 \mathrm{~cm} \mathrm{H}_{2} \mathrm{O}$ pro Liter Dehnung also eine Spannungszunahme von $15 \mathrm{~cm} \mathrm{H}_{2} \mathrm{O}$.

Über die Abhängigkeit von $\Sigma p_{\epsilon l}$ vom Dehnungszustand der Atemorgane liegen, wie ich erst nach Ableitung meiner Bestimmungsmethode sah, bereits Untersuchungen von Jaquet ${ }^{1}$ ) und Bernoulli ${ }^{2}$ ) vor. Die Messungen geschahen auch bei Muskelerschlaffung, indem die Versuchsperson in eine pneumatische Kammer gesetzt wurde, deren Kombination mit einem grossen maschinell angetriebenen Blasbalg rythmische Druckänderung der Kammerluft ermöglicht. Die Volumänderung des Thorax bei verschiedenen Druckänderungen werden spirometrisch gemessen. Diese Methode, welche die elastische Spannungsänderung der Atemorgane bei einer Änderung des Dehnungszustandes durch eine äussere Luftdruckänderung misst, sonst aber auf demselben Prinzip wie unsere Methode beruht, hat den Nachteil einer umfangreichen komplizierten Apparatur. Ferner scheint es mir viel leichter, bei abgeschlossenen Atemwegen und sistierter Atmung die Muskeln erschlaffen zu lassen, als dies möglich ist bei durch äussere Druckänderungen bewirkten passiven Atembewegungen.

Bernoulli ${ }^{3}$ ) nahm Messungen an sechs Versuchspersonen vor und erhielt ganz ähnliche, in den mittleren Partien annähernd gleichmässig gereigte, gegen die extremen Lagen zunehmend steilere

1) Arch. f. exper. Pathol. u. Pharmakol. 1908 Supplbd. S. 309-316.

2) Arch. f. exper. Pathol. u. Pharmakol. Bd. 66 S. 313-333. 1911.

3) Arch. f. exper. Pathol. u. Pharmakol. Bd.. 66 S. 321. 1911. 
Kurven, wie wir für unsern Fall finden. Aus Kurve III und $I V$; welche auch Männer von 28 Jabren betreffen, messe ich pro Liter Volumdehnung in dem mittleren 2 Liter umfassenden Dehnungsbereich eine $\Sigma p_{\epsilon l}$ - Änderung von 10,5 bezw. $9,5 \mathrm{~mm} \mathrm{Hg}$, entsprechend 14,3 bezw. $12,9 \mathrm{~cm} \mathrm{H}_{2} \mathrm{O}$. In unserem Fall fanden wir $14 \mathrm{~cm}_{2} \mathrm{O}$.

Die direkte Messung von $\Sigma p_{e l}$ verschiedener Dehnungszustände bei Muskelerschlaffung erfordert immerhin auch bei unserem Vorgehen eine ziemliche Übung der Versuchsperson. Ein orientierendes, vor allem für klinische $Z$ wecke genügend genaues indirektes Messverfahren, welches viel rascher und auch bei wenig intelligenten Patienten anwendbar ist, werden wir später angeben.

\section{Die elastische Spannung der Brusthöhlenumgebung.}

$\Sigma p_{\epsilon l}$ besteht aus den beiden Komponenten $p_{e l}$ thor und $p_{e l}$ pulm. Da wir den Gesamtwert wie auch die letztere Komponente (S. 421) kennen, ergibt sich aus der Gleichung $\Sigma p_{\epsilon l}=p_{\text {el prtm }}-p_{\epsilon l \text { thor }}$ durch Umstellung die Beziehung, nach welcher für die einzelnen Dehnungszustände $p_{\epsilon b \text { thor }} \mathrm{zu}$ berechnen ist.

$$
p_{\epsilon l \text { thor }}=p_{\epsilon l} \text { pulm }-\Sigma_{p_{\epsilon l}}
$$

Da es sich um den Ausdruck eines Kräfterleichgewichts handelt, entspricht der Gleichheit Entgegengesetztkeit der Vorzeichen, zum Beispiel bei Gleichgewichtslage $\Sigma_{p_{l l}}=0$ ist der Thoraxzug nach aussen gleich und entgegengesetzt dem Zug des Brustinhaltes nach innen.

In Fig. 2 sind die Linie für $p_{\text {el pulm }}(I I I)$ und die Kurven für $p_{\text {elthor }}(I$ und $I I$ ) eingezeichnet. Kollaps und Reserveluft nehmen wir für die Versuchsperson, deren vitale Kapazität (3,3 Liter), unter dem Mittel liegt, entsprechend kleiner als die Mittelwerte an: 0,55 bzw. 1,1 Liter. Die Kurven für $p_{\text {elthor }}$ bei verschiedener Körperstellung sind ähnlich denjenigen von $\Sigma p_{e l}$, aber flacher und tiefer gelegen. Sie zeigen entsprechend jenen einen geraden Verlauf im mittleren Dehnungsbereich. Bei Gleichgewichtslage sind sie gleichweit unterhalb der Abzcissenachse wie die Lungenelastizitätslinie oberhalb. Ihre Schnittpunkte mit der Abszissenachse, für aufrechte Haltung ca. ${ }^{2 / 3}$ Liter unter maximaler Inspiration, entsprechen der elastischen Gleichgewichtslage der Brusthöhlenwandung bei diesen verschiedenen Körperstellungen. Ihre zunehmende Steilheit gegen die extremen Lagen hin ist dadurch bedingt, dass hier neue Spannungsmomente auftreten, indem in den verschiedenen Gelenken des Thorax- 
skelettes die Bewegungsmöglichkeit erschöpft wird und eine elastische Dehnung der Gelenkkapseln und Bänder einsetzt, anderseits, indem die Weichteile des Thorax und exspiratorisch das Zwerclifell, inspiratorisch die Bauchdecken ihrer Dehnungsgrenze sich nähern. Die ræschere Spannungsänderung gegen die exspiratorische Grenzlage hin scheint mir dalin deutbar zu sein, dass hier das während der vorgehenden Exspirationsbewegung durch die Bauchpresse in die Brust-

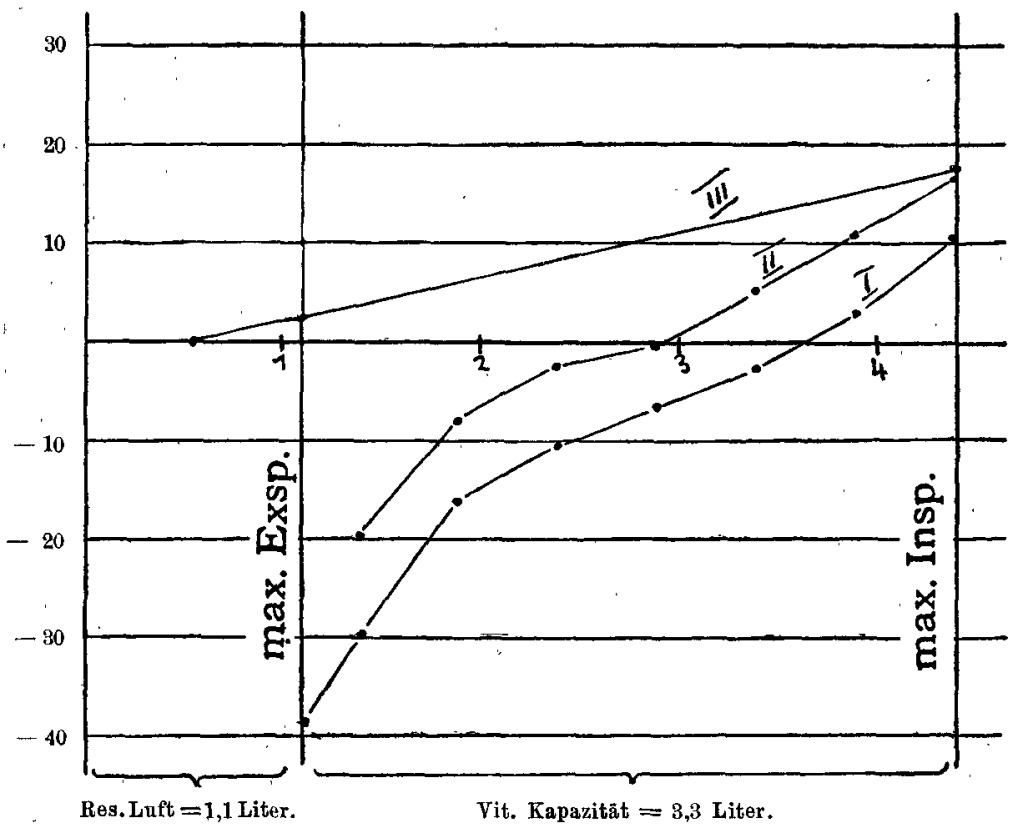

Fig. 2. Abszissenwerte $=$ Lungenluftrolum in Litern. Ordinatenwerte $=$ Druck in Zentimetern $\mathrm{H}_{2} \mathrm{O}$. $-I$ Kurve der elastischen Spannung in der Brusthöhlennmgebung im Sitzen. II Kurve im Liegen. III Linie der elastischen Retraktionskraft des Brustinhaltes.

höhle emporgetriebene Diaphragma rascher der Grenze der Dehnbarkeit sich nähert, als umgekehrt bei inspiratorischer Grenzlage die Bauchdecken angespannt werden.

Die verschiedene Lage der $p_{t t \text { thor }}$-Kurve bei verschiedener Stellung des Körpers ist, wie schon betont, durch die wechselnde Wirkung der Schweremomente bedingt. In aufrechter Haltung hat das Thoraxgewicht die Tendenz, den Thoraxbinnendruck zu erhöhen, der Gewichtszug des Bauchinbaltes (Leber usw.) wirkt umgekehrt im Sinne einer Druckverminderung. Wie das Höhersteigen der Kurve inı Liegen zejgt, überwiegt bei aufrechter Körperlage das letztere Moment. 
Ein weiteres Moment, welches auf die Lage der $p_{\text {el thor }}$-Kurve Einfluss hat, ist die Form der Brusthöhle. Wenn wie bei Muskelerschlaffung nur elastische Kräfte und Schweremomeute wirken, besitzt der Brustraum infolge innern und äussern Spannungsausgleiches diejenige Form, bei welcher an jeder Oberflächeneinheit die gleiche elastische Kraft wirkt: $p_{\epsilon l \text { thor }}=p_{\epsilon l \text { pulm }}+p_{u l v \text {. }}$. Wenn muskuläre Kräfte sich mitbeteiligen, ist infolge des Spannungsausgleiches zwar $p_{\text {musk }}$

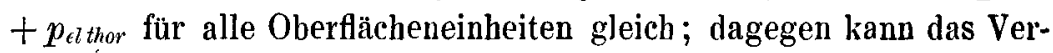
hältnis-der beiden Komponenten zueinander, an verschiedenen Stellen der Brusthöhlenwand verschieden sein, und es ist fraglich, ob der Mittelwert von $p_{c \text { thor dann }}$ gleichgross ist wie bei Muskelerschlaffung. Ferner kann die Aktion der inspiratorischen und exspiratorischen Muskulatur, da sie verschiedene Angriffspunkte haben, bei dem gleichen Dehnungszustand etwas verschiedene Brustböhlenform und damit Verschiedenheit der Verteilung von $p_{\text {elthor }}$ und vielleicht auch des Mittelwertes derselben bedingen. Wenn man zum Beispiel ausgehend von Gleichgewichtslage bei geschlossener Glottis maximal inspiratorische, nachher maximal exspiratorische Muskelkraft wirken lässt, so ändert dabei die Thoraxform in verschiedenem Sinn. Es tritt allerdinğs dảs eine Mal durch Dehnung der Lungenluft eine Erhöhung, das andere Mal eine Kompression und Verringerung des Dehnungszustandes ein, welche für unsere Versuchsperson nach Berechnung aus Gesamtvolumen und Druckänderung beidemal ca. 0,3 Liter beträgt. Um dieses Moment zu vermeiden, lässt man vor der inspiratorischen Anstrengung dieses Volumen ins Spirometer ausatmen, vor der exspiratorischen so viel einatmen. Auch dann ist woch eine Verschiedenheit der Thoraxform, vor allem in den unteren Abschnitten, zu beobachten. Bei in genannter Weise hergestelltem gleichgrossem Thoraxinnenraum ist der Umfang $4 \mathrm{~cm}$ unterhalb des Processus xiphoideus :

elastische Gleichgewichtslage . . . . . . $68,5 \mathrm{~cm}$ maximale inspiratorische Anstrengung . . . $71 \mathrm{~cm}$ maximale exspiratorische Anstrengung . . . . $68 \mathrm{~cm}$

Wie dieser Versuch zeigt, ist bei gleichem Dehnungszustand die Thoraxform bei Muskelanstrengung eine etwas andere als bei blossem Wirken elastischer Kräfte, und zwar ist sie bei inspiratorischer An. strengung mehr in inspiratorischem, bei exspiratorischer mehr in exspiratorischen Sinue verändert. Entsprechend ist anzunehmen,

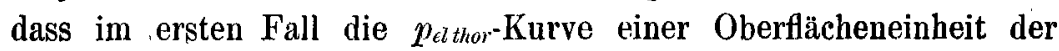


Thoraxinnenfläche nach oben, im zweiten Fall nach unten verschoben ist. Der Unterschied wird aber der geringen Formdifferenz entsprechend klein sein, und wenn auf der diaphragmalen Seite der Brusthöhle die Verhältnisse umgekehrt liegen, kann für den mittleren $p_{\text {el thor }}$-Wert die Differenz sogar versehwinden.

Die $p_{\epsilon l}$ thor -Kurve ist auch beeinflussbar durch Anbringung äusserer elastischer Momente. Das Anlegen einer elastischen Binde am Thorax verschiebt die $p_{\ell \text { th }}$ - Kurve nach oben, ungefähr ihrem Verlauf im Liegen entsprechend, ohne wesentlich ihre Neigung zu beeinflussen. Letzteres scheint darauf hinzuweisen, dass der Organismus die Form der Atmung den gegebenen Verhältnissen so anpasst, dass mit dem Liter Volumdehnung die geringste. Zunahme des Mittelwertes von $p_{t l}$ thor verbunden ist. Dieses Moment ist wahrscheinlich auch bestimmend für den Atemtypus. Wenn z. B. abdominell durch raumbeengende Organe (Gravidität, Tumoren, Meteorismus) oder durch beengende Kleider eine Volumänderung mit grosser elastischer Spannungszunahme verbunden ist, so wiegt kostaler Atemtypus vor. Die diaphragmale Atmung scheint mit geringerer Spannungszunahme verbunden zu sein, indem sie normalerweise vorherrseht. Wenn durch Altersveränderungen der Thorax starr wird, pflegt die kostale Atmung sogar weitgehend eingeschränkt zu werden, und weil die elastischen Kräfte abdominell im Gegenteil zum Thorax im Alter sich verringern, sehen wir beim Emphysem, obschon ein Teil der Brusthöhlenwandung starrer, d. h. fähiger zur inspiratorischen Speicherung elastischer Kräfte ist, trotzdem die Exspiration erschwert und nur unter Beiziehung muskulärer kräfte vor sich gehen, indem die vorherrsehende Bauchatmung bei den verringerten elastischen Spannungen im Abdomen des Emphysematikers pro Liter Volumdehnung unter geringerer $s p_{e l}$ - Änderung einhergeht und damit für eine passive Exspiration nachher geringere Kräfte zur Vterfügung stehen (S. 438).

\section{Die Verhältnisse bei maximaler Muskelanstrengung.}

\section{Die maximale inspiratorische und exspiratorische Kraft.}

Sie ist bei einem bestimmten Dehnungszustand messbar durch die alveoläre Druckdifferenz, welche bei abgeschlossenen Atemwegen unter maximaler Anspannung der inspiratorischen bzw. exspiratorischen Muskulatur erzeugt wird. 
Es ist zu berücksichtigen, dass inspiratorisch dabei die Thoraxluft gedehnt, exspiratorisch komprimiert wird. Wenn der Lungenluftgehalt im Ausgangszustand, bei Barometerstand $b, L$ Liter beträgt and eine Druckdifferenz $p_{a l n}$ erzengt wird, so ist noch dem Mari otteschen Gesetze das erreichte Volumen: $L^{\prime}=I \frac{b}{b \mp p_{a l v}}$, wobei inspiratorisch das obere, exspiratorisch das untere Vorzeichen gilt. ( $b$ ist für Täbingen ca. $730 \mathrm{~mm} \mathrm{Hg.)} \mathrm{Die} \mathrm{gemessene} \mathrm{inspiratorische} \mathrm{bzw.}$ exspiratorische Kraft ist auf den so korrigierten Dehnungszustand zu beziehen. Da $p_{\text {alv }}$ bis $1 / 7$ Atmosphären, $L$ bis 5 Liter betragen kann, darf diese Korrektur nicht unterlassen werden.

Für die gleiche Versuchsperson wie oben, im Sitzen und ausgehend von derselben, je $1 / 2$ Liter auseinanderliegenden Dehnungszuständen erhalten wir folgende Werte:

\begin{tabular}{|c|c|c|c|c|c|c|c|c|}
\hline & \multicolumn{8}{|c|}{ Exspiration in Litern } \\
\hline & 3,3 & 3 & 2,5 & 2 & 1,5 & 1 & 0,5 & 0 \\
\hline Lungenluftvolum . . & 1,1 & 1,4 & 1,9 & 2,4 & 2,9 & 3,4 & 3,9 & 4,4 \\
\hline $\begin{array}{l}\text { Max. Exspirationsdruck } \\
\text { in Zentimetern } \mathrm{H}_{2} \mathrm{O} \text {. } \\
\text { Korrig. Bezugsvolumen }\end{array}$ & $\begin{array}{l}0 \\
1,1\end{array}$ & $\begin{array}{l}48 \\
1,33\end{array}$ & $\begin{array}{l}86 \\
1,75\end{array}$ & $\begin{array}{l}106 \\
\quad 2,17\end{array}$ & $\stackrel{116}{2,6}$ & $\begin{array}{r}124 \\
3,0\end{array}$ & $\begin{array}{l}132 \\
3,45\end{array}$ & $\begin{array}{r}142 \\
3,85\end{array}$ \\
\hline $\begin{array}{l}\text { Max. Inspirationsdruck } \\
\text { in Zentimetern } \mathrm{H}_{2} \mathrm{O} \text {. } \\
\text { Korrig. Bezugsvolumen }\end{array}$ & $\begin{array}{r}-138 \\
1,27\end{array}$ & $\begin{array}{r}-133 \\
1,61\end{array}$ & $\begin{array}{r}-122 \\
2,17\end{array}$ & -111 & $\begin{array}{r}-92,5 \\
3,2\end{array}$ & $\begin{array}{l}-68 \\
3,65\end{array}$ & $\begin{array}{r}-43,5 \\
4,1\end{array}$ & $\begin{array}{l}0 \\
4,4\end{array}$ \\
\hline
\end{tabular}

Die Exspirationsdrucke wurden mit dem nach Zentimeter $\mathrm{H}_{2} \mathrm{O}$ geeichten Manometer von Recklinghausen gemessen, die Inspirationsdrucke mit einem offenen Quecksilbermanometer und in Zentimeter $\mathrm{H}_{2} \mathrm{O}$ umgerechnet. (Technik der Messung, S. 435-436.)

In Fig. 3 sind über den Dehnungszuständen als Abszissen diese Druckwerte als Ordinaten aufgezeichnet und durch Kurven verbunden. Die nach oben liegende Kurve des maximalen Exspirationsdruckes $(I)$ hat ihren Höchstwert bei maximaler Inspirationslage, von der aus eine nicht unbeträchtliche Exspirationsbewegung erfolgt (ca. 0,55 Liter), bis der Überdruck der Lungenluft der exspiratorischen Kraft das Gleichgewicht bält. Bis zu einem Dehnungszustand, der ca. 1/2 Liter oberhalb der maximalen Exspiration liegt, sinkt die Exspirationskraft annähernd gleichmässig auf zirka die Hälfte ihres Anfangswertes, um dann von hier an steil bis auf Null bei maximaler Exspirationslage zu sinken.

Die nach unten liegende Kurve der maximalen inspiratorischen Kraft (II) zeigt einen ähnlichen, nur gleichmässig gebogenen Ver- 
lauf in umgekehrter Richtung, ca. $3 / 4$ Liter unterhalb der maximalen Inspirationslage ist ungefähr noch die Hälfte des bei maximaler Exspirationslage messbaren höchsten Wertes vorhanden.

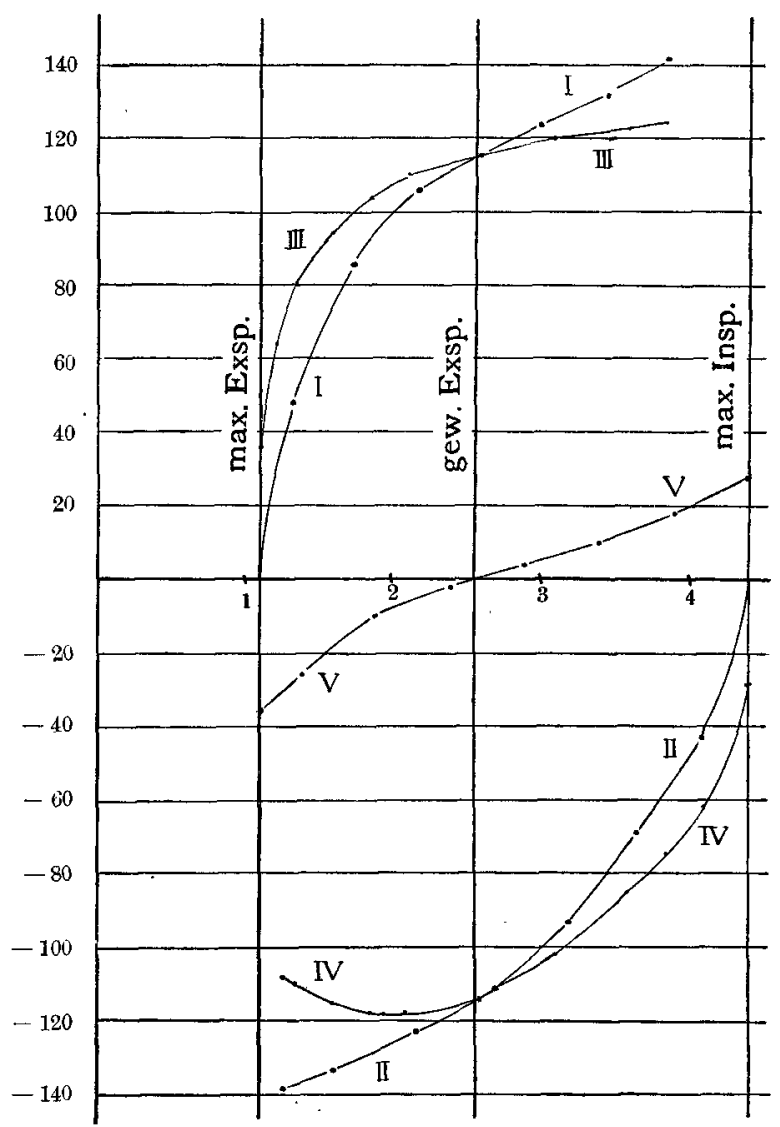

Fig. 3. Abszissenwerte $=$ Lungenluftvolumen in Litern. Ordinatenwerte $=$ Druck in Zentimetern $\mathrm{H}_{2} \mathrm{O}$. $-I$ Kurve des maximalen Exspirationsdruckes. II Kurve des maximalen Inspirationsdruckes. III Kurve der maximalen exspiratorischen Muskelkraft. $1 V$ Kurve der maximalen inspiratorischen Muskelkraft. $\quad V$ Kurve der elastischen Spannungsresultante.

\section{Die maximale inspiratorische und exspiratorische Muskelkraft.}

Da $p_{\text {musk }}=\Sigma p_{e l}+p_{a l v}$ ist und wir für die verschiedenen Dehnungszustände sowohl $\Sigma_{p_{e l}}$ wie den inspiratorischen und exspiratorischen Hochstwert von $p_{a l v}$ kennen, können wir durch Addition, unter Beräcksichtigung der Vorzeichen, die Werte der maximalen inspiratorischen und exspiratorischen Muskelkraft der verschiedenen Dehnungs- 
zustände berechnen. Unter normalen Verbältnissen ist die $\Sigma_{p_{\ell l}}$ Kurve für diese inspiratorischen und exspiratorischen Zustände, wie wir saben, wahrscheinlich wenig verschieden von derjenigen bei Muskelerschlaffung, die der Messung zugänglich ist (S. 431). In Fig. 3 ist diese Kurve nochmals eingezeichnet $(V)$. Unterhalb der Gleichgewichtslage wirkt $\Sigma_{p_{t l}}$ inspiratorisch. Die Ordinatenwerte sind hier bei der inspiratorischen Kurve von $p_{a t n}$ zu subtrahieren, bei der exspiratorischen $p_{a l v}$-Kurve $\mathrm{zu}$ addieren, um die $p_{m u s k}$ Werte zu erhalten. Oberhalb der Gleichgewichtslage sind die Verhältnisse umgekebrt. Bei Gleichgewichtslage ist $\Sigma_{e l}=0$ und $p_{m u s k i}=p_{a l v}$.

Die Kurve der maximalen exspiratorischen Muskelkraft (III), die wenig geneigt verläuft und gegen die maximale Exspiration hin plötzlich abfällt, zu einem Wert der gleich und entgegéngesetzt dem extremen exspiratorischen Wert von $\Sigma_{p_{c l}}$ ist, ist durch die Abnahme der muskulären Kraft bei zunehmender Muskelverkürzung zu deuten. Vor allem werden mit dem exspiratorischen Sinken des Thorax für die Bauchmuskulatur die Bedingungen der Kraftentfaltung zunehmend ungünstiger.

Die Kurve der maximalen irspiratorischen muskulären Kraftentwicklung $(I V)$ verläuft annähernd umgekehrt wie die der exspiratorischen Kraft. Auch ihr Verlauf ist wohl bedingt durch die Abnahme der Kraftleistung mit zunehmender Muskelverkürzung. Da ferner die Brusthöblenoberfläche inspiratorisch wächst, so geschieht die Abnahme der Kraft pro Oberflächeneinheit gegen die maximale Inspirationslage hin hier rascher als die Abnahme der exspiratorischen Kraft gegen maximale Exspiration hin. Dort ist durch die exspiratorisebe Verkleinerung der Brusthöhlenoberfläche die Kraftabnahme durch Muskelverkürzung zunächst fast kompensiert, die Kurve ist wenig geneigt und fällt dann mit einer Biegung plötzlich ab. Hier wirkt die Oberflächenzunahme, in gleichem Sinn wie die Muskelverkürzung, bei fortschreitender Inspiration verkleinernd auf $p_{\text {must }}$ der Oberflächeneinheit; die Kurve fällt gleichmåssig gekrümmt gegen die maximale Inspiration hin, wo sie einen Wert erreicht, der gleich und entgegengesetzt dem extrem inspiratorischen Wert von $\Sigma_{p_{e l}}$ ist. Der wichtigste Inspirationsmuskel, das Zwerehfell, zeigt hinsichtlich der Verkürzung im Verlaufe der Inspiration besondere Verhältnisse. Einerseits würde die inspiratorische Senkung des Centrum tendineum eine Verkürzung des Abstandes zwischen Origo und Insertio der Muskelbündel bedingen, anderseits die 
inspiratorische Erweiterung der unteren Thoraxapertur eine Verlängerung. Da eine von maximaler Exspiration ausgehende Inspirationsbewegung zunächst mit einer rapiden Erweiterung der unteren Thoraxapertur einsetzt, ist der Anstieg den die Kurve der maximalen inspiratorischen Muskelkraft hier zeigt, so zu deuten, dass zunächst der verlängernde Einfluss überwiegt.

\section{Indirekte Messung der elastischen Kräfte und die Verhältnisse beim Emphysem.}

Die maximale exspiratorische Muskelkraft zeigt zwischen maximalem Inspirationszustand und einem Dehnungszustand, der ca. 1/2 Liter oberhalb der maximalen Exspiration liegt, eine annähernd gleichmässige Abnahme, die in gleichem Sinn erfolgt wie die Änderung der elastischen Retraktionskraft der Respirationsorgane: $\Sigma p_{t \ell}$. Die Änderung von $\Sigma p_{e l}$ in diesem Bereich ist ferner bedeutend grösser, in unserem Fall z. B. ca. doppelt so gross wie die der Muskelkraft. Die Änderung der maximalen Exspirationskraft ist entsprechend in diesem Dehnungsbereich hauptsächlich abhängig von

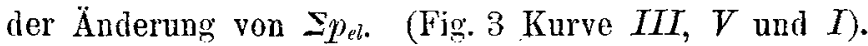

Wenn wir die maximale Exspirationskraft für die beiden gejannten Debnungszustände bestimmen $\left(p_{t t v_{1}}, p_{a t v_{2}}\right)$ und die Differenz beider Werte durch die vitale Kapazität $(C)$ teilen, erhalten wir einen rohen Relativwert für die Änderungsgrösse der elastischen Retraktionskraft der Respirationsorgane pro Liter Dehnung $\left(k_{\ell}\right)$

$$
k_{c b}=\frac{p_{a v_{1}}-p_{a l v_{2}}}{C} .
$$

In folgender Tabelle sind eine Anzahl derartiger Bestimmungen bei Männern mit gesunden Respirationsorganen zusammengestellt. Die Messung der vitalen Kapazität und der Druckwerte erfolgte stets drei- bis viermal. Die letzteren sind mit einem offenen Hg-Manometer bestimmt, und zwar wurde als richtiger Wert nicht der höchste Punkt, zu dem die Hg-Säule für einen Moment emporgetrieben wird (Schleuderung), sondern der höchste Punkt, auf dem sie nachher einige Sekunden oder länger verharrt, betrachtet. Die Schlenderung wurde meist durch langsam gesteigerte Druckentfaltung üherhaupt vermieden. Die Messung geschah vom Mund aus. Als Mundstück wurden stumpfwinklig abgebogene Trinkröhrchen verwendet, die ca. $3-4 \mathrm{~cm}$ tief in die Mundhöhle eingeführt wurden, um das Pressen vom Mund aus zu vermeiden. Wenn man zuerst die Spirometerbestimmung vornimmt, begreifen auch wenig intelligente Leute rasch, dass sie von der Lunge her pressen sollen. Für die Berechnung von $k_{c l}$ sind die höchsten bestimmten Werte der vitalen Kapazität und des maximalen Exspirationsdruckes in maximaler 
Inspirationsstellung zu benutzen. Für die Bestimmung des maximalen Exspirationsdruckes nahe der maximalen Exspirationsstellung liess ich zuerst vollständig exspirieren, dann möglichst wenig, ungefähr einem gewöhnlichen Atemzug entsprechend, inspirieren und nun ins Manometer den Druck entfalten. Es wurden hier meist vier bis sechs Messungen vorgenommen, die ersten zwei weggelassen und von den übrigen, die meist wenig streuten, das Mittel genommen. Die ganze Untersuchung erfordert mit Ruhepansen 10-15 Minuten. Schwierigkeiten ergeben sich nur bei hypochondrischen Neurasthenikern, die sich ängstlich hüten, ihre ganze Exspirationskraft zu entfalten. Bis jetzt sind mir nur drei solche Versager vorgekommen. Diese drei Versuche sind ausgeschaltet worden, während sonst alle bisherigen Versuche im folgenden zusammengestellt sind.

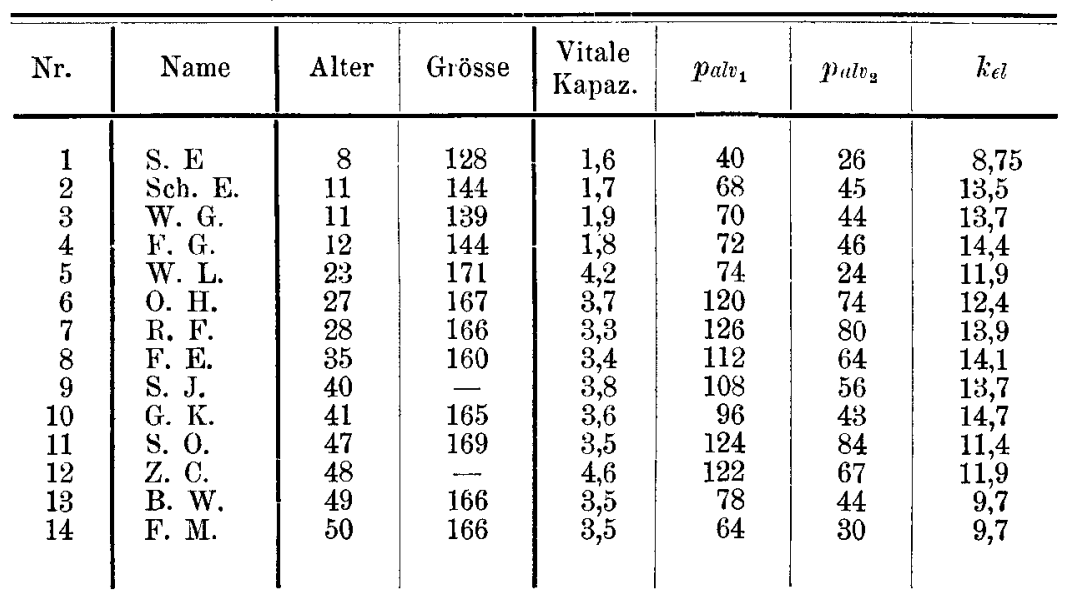

Ein Anteil von $p_{a l v_{1}}-p_{n l v_{2}}$ wird durch muskuläre Kräfte dargestellt, und es ist für die Verwendbarkeit der Formel wichtig, ob Änderungen der muskulären Kräfte das Ergebnis beeinflussen. Fall 7 ist die gleiche Versuchsperson wie oben in Abschnitt B und C. Die dortigen Messungen lagen hier ca. 4 Wochen zurück. Durch dauernde Übung hat inzwischen die Versuchsperson ihre exspiratorische Kraftentfaltung ca. um 20\% erhöht. Wenn wir dort (S. 433) aus der Kurve die Werte von $p_{a v_{1}}$ und $p_{a l v_{2}}\left(1_{1}\right.$ Liter obermaximaler Exspiration) ablesen und in Millimeter Hg umrechnen, erbalten wir $k_{\epsilon l}=\frac{104,3-57,3}{3,3}=14,2$ gegenüber 13,9 hier. Ein umgekehrter Fall ist Nr. 5 Patient ist in der Rekonvaleszenz von einer einige Monate zurückliegenden Magenblutung und zeigt, infolge noch verringerter muskulärer Leistungsfähigkeit, für sein Alter sehr niedrige Werte von $p_{a l v_{1}}$ und $p_{a l v_{2}}$. Der $k_{\epsilon l}$-Wert ist etwas niedrig, schliesst sich aber gut der übrigen Reihe an. Weitgehende Änderungen der muskulären Kräfte sind, wie diese zwei Beispiele zeigen, von kaum zu berücksichtigendem Einfluss auf die Grösse von $k_{e l}$ Dieser Wert muss also im wesentlichen nur abhängen von der elastischen Kraftänderung pro Liter Dehnung, und Verschiedenheiten von $k_{t l}$ werden Verschiedenheiten der letzteren entsprechen. 
Die hier mit in Millimeter Hg.Säule gemessenen Drucken, berechneten Relativwerte, entsprechen ziemlich genau den absoluten Werten in Zentimeter $\mathrm{H}_{2} \mathrm{O}$. (Fall $7: k_{\varepsilon l} 13,9$ und 14,2 ; absoluter Wert [S. 425] $k_{\epsilon l}=14 \mathrm{~cm} \mathrm{H}_{2} \mathrm{O}$.)

Wie die Versuchsreihe zeigt, ergibt diese Bestimmungsmethode für die Zunahme der elastischen Kraft der Respirationsorgane pro Liter Dehnung bei gesunden Individuen wenig verschiedene Werte. Besonders nahe liegen die $k_{e l}$-Werte von Fall 2-10, für Männer zwischen 11-41 Jahren. Das Mittel dieser neun Bestimmungen ist: 13,6; der niedrigste und höchste Wert : 11,9 und 14,7.

Im ersten Dezennium, wo der Thorax noch leicht deformierbar ist, haben wir geringere Werte zu erwarten. Fall $1 ; 8 J, k_{t l}=8,75$.

Die Werte der zweiten Hälfte des 5. Dezenniums liegen teilweise deutlich unterhalb der Werte früherer Lebensalter. Diese Erscheinung, welche mit beginnenden Altersveränderungen der Respirationsorgane in Zusammenhang zu bringen ist, leitet über $\mathbf{z u}$ den Verhältnissen, wie wir sie beim Emphysem finden:

18 Fälle von typischem Emphysem mit Thoraxstarre, welche in gleicher Weise wie oben vermessen wurden, geben folgende Mittel werte:

\begin{tabular}{c|c|c|c}
\hline Vitale Kapazität & $p_{a l_{1}}$ & $p_{a l v_{\mathbf{2}}}$ & $k_{\varepsilon l}$ \\
\hline 2,8 & 68,9 & 49,1 & 7,1
\end{tabular}

Die einzelnen $k_{\epsilon l}$-Werte verteilen sich folgendermaassen:

\begin{tabular}{l|c|c|c|c|c|c|c|c|c}
\hline & \multicolumn{6}{|c}{$k_{\epsilon l}=$} \\
\cline { 2 - 7 } & $3-5$ & $5-6$ & $6-7$ & $7-8$ & $8-9$ & $9-10$ & $10-12$ \\
\hline Zahl der Fálle \\
Davon acht Fälle mit $C j \dot{3}$ Liter. & 1 & 3 & 5 & 3 & 4 & 1 & 1 \\
\hline
\end{tabular}

Dass das Ergebnis nicht abhängt von der beim Emphysem oft vorhandenen Verringerung der vitalen Kapazität zeigen die zuletzt hervorgehobenen acht Fälle, deren vitale Kapazität zwischen 3,1 bis 3,8 Liter liegt.

Das Emphysem ist für die Atemphysiologie von Interesse, weil hier, trotzdem der morphologische Rahmen der Respirationsorgane weitgehend sich verschoben hat und fast alle Momente, die normalerweise für die Atemmechanik wichtig sind, quantitativ geändert haben, doch das gleiche Beziehungsgesetz der Atemkräfte gilt. 
Wie unsere Untersuchungsreihe beweist, ist beim Emphysematiker die Änderung der elastischen Kraft pro Liter Volumänderung der Respirationsorgane gegenüher dem Normalen bedeutend verringert, auf die Hälfte bis ein Drittel. Wenn die Atemorgane um ein bestimmtes Volumen von der Gleichgewichtslage entfernt sind, stehen hier für die Exspiration entsprechend geringere Kräfte zur Verfügung. Es entspricht diese Feststellung der Beobachtung, dass die Exspiration des Emphysematikers sehr erschwert ist und meist unter Beiziehung muskulärer Kräfte erfolgt.

Dieser wie mir scheint sichergestellte Befund steht nur scheinbar im Widerspruch mit der Thoraxstarre des Emphysematikers. Die hier in höherem Maasse vorhandenen elastischen Momente sind von geringem Einfluss, weil die thorakale Atmung eingeschränkt ist (S. 431), anderseits sind für die vorherrschende abdominelle Atmung die elastischen Widerstände herabgesetzt, teils durch Alterserschlaffung der Bauchwände, teils, weil durch die meist vorhandene, oft sogar sehr bedeutende, Vorwärtsbiegung der Brustwirbelsäule der Thorax dem Becken sich nähert. Das Abdomen zeigt beim Emphysematiker entsprechend eine eigenartige Oberflächengestaltung. Besonders charakteristisch ist eine $2-4 \mathrm{~cm}$ oberhalb des Nabels quer verlaufende Furche, welche am ausgeprägtesten im Stehen und bei stärker kyphotischen Emphysematikern zu beobachten ist. Ein Anteil an der Verringerung von $l_{c l}$ ist beim Emphysematiker auch durch die elastische Erschlaffung des Lungengewebes bedingt.

\section{Die an der Gesamtoberfläche der Brusthöhle wirkenden Kräfte.}

Für den Inhalt des Brustraumes der Versuchsperson bei gewöhnlicher Exspirationsstellung erhalten wir nach der in nachstehender Arbeit über: "Bestimmung des Inhaltes und der Oberfläche des Brustraumes beim Lebenden " entwickelten Methode einen Wert von 5,345 Liter. Die Brusthöhlenoberfläche bestimmt sich zu 18,54 qdm, für einen anderen Dehnungszustand $J$ zu $6,065 \cdot J^{2 / 3}$. Die Reserveluft der Versuchsperson beträgt 1,5 Liter. Für die oben mehrfach benützten, je ${ }^{1 / 2}$ Liter auseinanderliegenden Dehnungszustände erhalten wir folgende Übersicht: 


\begin{tabular}{l|r|r|r|r|r|r|r|r}
\hline & \multicolumn{6}{|c|}{ Lungenluftvolumen in Litern } \\
\cline { 2 - 8 } & 1,1 & 1,4 & 1,9 & 2,4 & 2,9 & 3,4 & 3,9 & 4,4 \\
\hline Brusthöhleninhalt: Liter. & 3,85 & 4,15 & 4,65 & 5,15 & 5,65 & 6,15 & 6,65 & 7,15 \\
Brusthöhlenoberflache: qdm & 14,86 & 15,63 & 16,9 & 18,1 & 19,22 & 20,35 & 21,44 & 22,5
\end{tabular}

Während sich beim Übergang von maximaler Exspiration zu maximaler Inspiration das Brusthöhlenvolumen der Versuchsperson nahezu verdoppelt, wächst dabei die Brusthöhlenoberfläche auf etwa das $1 \frac{1}{2}$ fache des Anfangswertes. Pro Liter Dehnung vergrössert sich die Brusthöhlenoberfläche je nach dem Ausgangszustand um $2^{1 / 2--2}$ qdin.

Um die Gesamtkräfte zu erhalten, sind die oben angegebenen Werte der Kräfte am qcm Oberfäche $p$, für jeden Dehnungszustand mit der entsprechenden Oberflächengrösse $O$ zu multiplizieren. Für $p$ in $\mathrm{cm} \mathrm{H}_{2} \mathrm{O}$ und $O$ in $\mathrm{qdm}$ ist die Gesamtkraft in Kilogramm:

$$
\text { Ges. } K \boldsymbol{r} .=0,1 \cdot p \cdot O \mathrm{~kg} \text {. }
$$

Wie mir durch die früheren Ausführungen genügend gesichert erscheint, darf für die im Innern des Thorax wirkenden Kräfte: $p=p_{\epsilon l p u l m}+p_{u l v}$, die auch am Mediastinum angreifen, die ganze Brusthöhlenoberfläche als Wirkungsfläche betrachtet werden. Das gleiche gilt auch für die äussere Kraftresultante: $x=p_{\text {musk }}+p_{\text {el thor }}$. Als Anteil der dehnenden Kraft $p$ sind an jeder Oberflächeneinheit die dorthin fortgeleiteten, senkrecht zur Oberfläche ansetzenden Komponenten der muskulären und elastischen Kräfte zu betrachten. $p_{e l t h o r}$ massen wir bei Muskelerschlaffung und sahen, dass bei Muskelanstrengung der Mittelwert von pelthor nur wenig versebieden von ersterem Wert sein kann; der übrige Anteil von $p$ muss also durch muskuläre Kraft geleistet werden, und die muskuläre Gesamtkraft wäre entsprechend als $O \cdot\left(p-p_{t \prime}\right.$ thor $)$ anzusehen.

Wenn wir zunächst nicht berücksichtigen, dass die muskulären Kräfte in der direkten Spannrichtung der Muskeln je nach den Hebelverhältnissen verschieden sein können von der von ihnen auf die Brusthöhlenoberfläche ausgeübten Kraft, so scheint noch ein weiterer Grund zu bestehen, der einen solchen Unterschied bedingen könnte.

Die Bewegung der einzelnen Brusthöhlen-Wandungsabschnitte bei der Atmung ist von verschiedener Grösse. Es besteht die Möglichkeit, dass die Muskulatur nur an den hauptsächlich bewegten 
Abschnitten wirkt und die übrigen durch innern Druckausgleich auf die gleiche Spannung gebracht werden, ähnlich wie in einer hydraulischen Presse, von einem kleineren bewegten Wandungsteil aus, die gesamte Oberfläche des mit Flüssigkeit gefüllten Binnenraumes der Presse infolge der Druckfortpflanzung auf den gleichen Druck gebracht wird, wie er an der kleinen bewegten Fläche wirkt. So könnte zum Beispiel bei maximaler exspiratorischer Druckentfaltung die Bauchpresse, das Diaphragma in die Brusthöble emportreibend, zugleich durch die Steigerung des thorakalen Binnendruckes auch die Brustkorbinnenfläche indirekt auf gleich bohe Spannung bringen. In diesem Fall wäre dann im wesentlichen nur das Zwerchfell als eigentliche Wirkungsfläche von $p_{\text {musk }}$ zu betrachten.

Wie mir scheint, darf dieser Vergleich mit der hydraulischen Presse nicht auf die Respirationsorgane angewandt werden. Das Thoraxskelett ist ein in allen seinen Teilen bewegliches System. Sogar die Brustwirbelsäule, die relativ die geringste Bewegungsmöglichkeit besitzt, zeigt sagittal und frontal eine nicht unbeträchtliche Biegungsfähigkeit. Den von vorn her sagittal angreifenden Zug oder Druckmomenten des Brusthöhleninhaltes gegenüber verhält sich die Brustwirbelsäule nicht passiv, so dass sie entsprechend der Zug- oder Druckrichtung ihre Form anpassen würde, sondern wir sehen im Gegenteil inspiratorisch, wo der Zug des Brustinhaltes nach vorn wächst, eine zunehmende Gradstreckung (der arrector trunci ist entsprechend als ein schon normalerweise zum Teil inspiratorisch tätiger Muskel anzusehen), bei exspiratorischer Kraftanstrengung umgekehrt eine Vorwärtsbiegung der Brustwirbelsäule. Da sogar dieser am wenigsten bewegliche Teil des Brustkorbes bei der Atmung sich aktiv verhält, scheint mir, dass auch für $p_{\text {musk }}$ die Gesamtoberfläche der Brusthöhle als Bezugsfläche anzusehen ist.

Wie weit infolge der Hebelverhältnisse die gesamte Spannung in der Zugrichtung der einzelnen Atmungsmuskeln verschieden ist von der auf die Brustböhlenoberfläche wirkenden, muskulär geleisteten Gesamtkraft, wäre nur durch eingehende Untersuchung der Hebelverhältnisse zu entscheiden. Für die spätere Untersuchung über die Atmungsarbeit ist ein allenfalls statisch vohandener Unterschied der zwei Kraftsummen nebensächlich, indem die Arbeitsgrösse beiderseits gleich sein muss.

Fig. 4 zeigt die Grösse der Gesamtkräfte in Kilogrammen bei den verschiedenen Dehnungszuständen. Die einzelnen Ordinaten- 
werte sind aus Fig. 3 (S. 433) durch Multiplikation mit der den einzelnen Dehnungszuständen entsprechenden Oberflächengrössen der Brusthöhle berechnet.

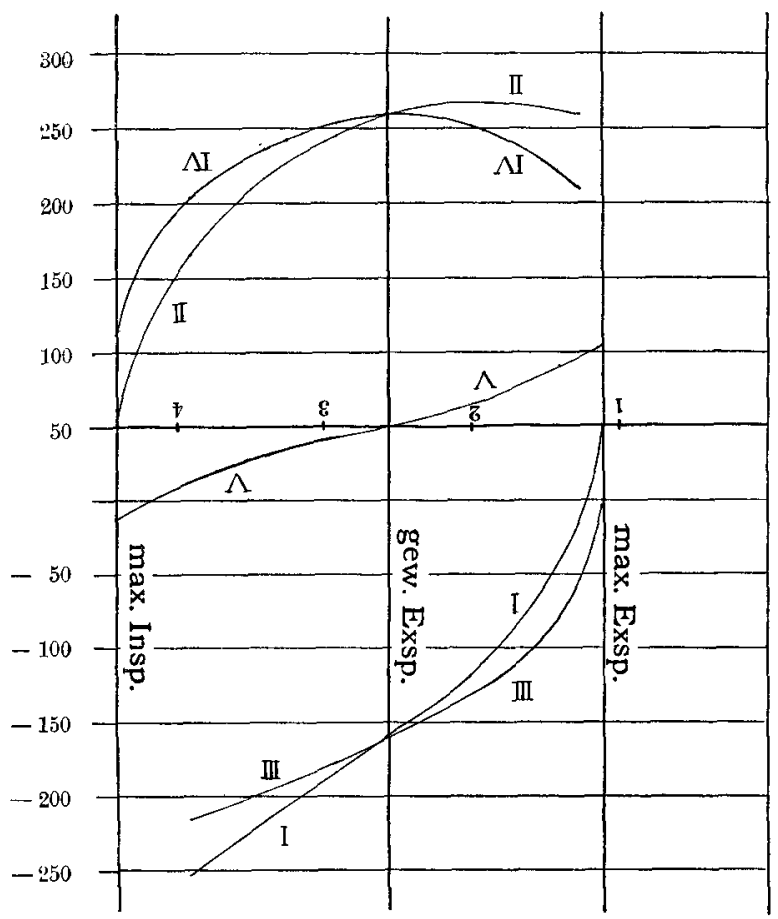

Fig. 4. Abszissenwerte $=$ Lungenluftvolum in Litern. Ordinatenwerte $=$ gesamte, an der Brusthöhlenoberfläche wirkeude Kraft in Kilogramm, nach oben exspiratorischer Druck, nach unten inspiratorischer Zug. - I Kurve der maximalen exspiratorischen Gesamtkraft. $I I$ Kurve der maximalen inspiratorischen Gesamtkraft. III Kurve der maximalen exspiratorischen muskulären Gesamtkraft. IV Kurve der maximalen inspiratorischen muskulären Gesamtkraft. $V$ Kurve der elastischen Gesamtkraft.

Die IKurven verlaufen ähnlich wie die der Kräfte an der Oberflächeneinheit. Noch auffallender als in Fig. 3 ist hier die Zurückbiegung der Kurve der maximalen inspiratorischen Muskelkraft, die wir oben (S. 434) auf die besonderen Verhältnisse des Zwerchfells zurückführten. Auch die Kurve des maximalen inspiratorischen Gesamtdruckes zeigt hier eine leichte Rückbiegung. Die höchste inspiratorische Kraftentfaltung bei unserer Versuchsperson: $220 \mathrm{~kg}$, ist etwas unterhalb der Gleichgewichtslage möglich, die der inspiratorischen Muskulatur ist bei Gleichgewichtslage $210 \mathrm{~kg}$. 
Die höchste exspiratorische Kraftentwicklung findet sich nahe der maximalen Inspirationsstellung. Der exspiratorische alveoläre Gesamtdruck an der Brusthöhlenoberfläche beträgt hier für die Versuchsperson $305 \mathrm{~kg}$, die muskulär bedingte Gesamtkraft $266 \mathrm{~kg}$. Die muskuläre Gesamtkraft bei Gleichgewichtslage ist für die Versuchsperson exspiratorisch gleichgross wie inspiratorisch.

Die elastischen Kräfte allein üben bei maximaler Inspirationsstellung einen Druck von $63 \mathrm{~kg}$, bei maximaler Exspirationsstellung einen Zug von $53,5 \mathrm{~kg}$ an der Brusthöhlenoberfläche aus.

Da unsere Versuchsperson eine unter dem Mittel liegende vitale Kapazität und mässig kräftig entwickelte Muskulatur besitzt, dürften für Erwachsene alle Werte durchschnittlich noch höher liegen.

Zum Vergleich mit diesen Ergebnissen diene die Schätzung von Donde $\mathrm{rs}^{1}$ ), dass die maximale Kraftleistung der Atemmuskulatur über $200 \mathrm{~kg}$ betrage, und die Angabe von Fick ${ }^{1}$ ), dass die Musculi intercostales externi allein einer Kraftentwicklung von ca. $94 \mathrm{~kg}$ fähig sind.

\section{Zusammenfassung.}

1. Die Atemkräfte sind messbar durch die Druckdifferenz, welcher sie Gleichgewicht halten. Der so bestimmte Manometerwert entspricht der Kraftg̣oösse an der Oberflächeneinheit des Brustraumes.

2. Der Zusammenhang der Atemkräfte unter statischen Verhältnissen für irgendeine Flächeneinheit der Brusthöhlenoberfläche ist in folgender Gleichung darstellbar:

$$
p=p_{\text {musk }}+p_{\text {cl thar }}=p_{\text {el putin }}+p_{\text {alv }}
$$

$p=$ Resultante der äusseren Kräfte (dehnende Kraft) $=$ Resultante der inneren Kräfte (Retraktionskraft des Brusthöhleninhaltes).

$p_{m u s k}=$ senkrecht zur Brusthöhlenoberfläche wirkende muskulär geleistete Kraft.

$p_{c t \text { thor }}=$ senkrecht zur Brusthöhlenoberfiäche wirkende, durch elastische und Schweremomente in der Brusthöhlenumgebung bedingte Kraft.

$p_{\epsilon l}$ puln $=$ elastisehe Retraktionskraft der Lungen pro Quadratzentimeter Oberfläche.

$p_{a b v}=$ alveoläre Druckdifferenz.

1) R. du Bois-Reymond, Mechanik der Atmung. Ergebn. d. Physiol. v. Asher-Spiro Bd. 1 (2) S. 402. 1902. 
3. Der Brusthöhleninhalt bringt wie eine ideale Flüssigkeit alle im Innern und an verschiedenen Stellen seiner Oberfläche wirkenden Kräfte unter sich zum Ausgleich: innerer und äusserer Spannungsausgleich. (Nur zwei kleinere Volumanteile des Brustraumes besitzen eine Sonderstellung. a) Die medial oberhalb des Hilus gelegenen Lungenabschnitte bei tiefer Inspiration und Exspiration. b) Die Bluträame des Mediastinums infolge der Herzmuskeltätigkeit.)

4. Die elastische Spannungsresultante $\left(\Sigma_{c l}=p_{c l}\right.$ prtm $\left.-p_{e l \text { thor }}\right)$ ist direkt messbar durch die bei Muskelerschaffung von ihr bedingte alveoläre Druckdifferenz.

Die $\Sigma_{c l}$-Werte sind abhängig vom Dehnungszustand der Atemorgane (Fig. 1, Kurve $I$ ), und zwar ist im mittleren, hauptsächlich für die Atmung wichtigen, 2 Liter umfassenden Dehnungsbereich die elastische Spannungsresultante eine lineare Funktion des Lungenluftgehaltes, das heisst die Änderung der elastischen Spannung erfolgt hier proportional der Volumänderung ( $Q$ in Litern):

$$
\Sigma p_{\epsilon l}=\Sigma p_{\epsilon l} \pm k_{c l} \cdot Q \text {. }
$$

Die $\Sigma p_{\ell \ell}$-̈̈nderung pro Liter Volumänderung beträgt hier für die Versuchsperson $k_{\epsilon l}=14 \mathrm{~cm}$ Wassersäule.

Die $\Sigma_{p_{c l}}$ Werte sind ferner abhängig von der Körperlage, indem dabei die Schweremomente je nachdem in verschiedener Richtung

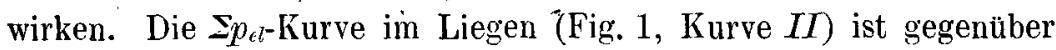
der im Sitzen nach aufwärts verschoben, ohne dass sie ihre Neigung ändert $\left(k_{e l}=14 \mathrm{~cm} \mathrm{H}_{2} \mathrm{O}\right)$.

Die Anbringung einer elastischen Binde am Thorax erhöht alle $\Sigma p_{\epsilon l}$-Werte, auch hier, ohne dass die Neiqung der Kurve wesentlich ändert.

5. Ein Relativwert der $\Sigma_{c c}$-Änderung pro Liter Dehnungsänderung der Respirationsorgane $\left(k_{e l}\right)$ wird indirekt erhalten durch Messung des maximalen Exspirationsdruckes in maximaler Inspirationsstellung $\left(p_{a v_{1}}\right)$ und etwa $1 / 2$ Liter oberhalb maximaler Exspirationsstellung $\left(p_{a v_{2}}^{\prime}\right)$ und Teilung der Differenz beider Werte durch die vitale Kapazität $(C)$ :

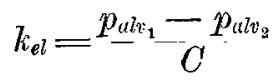

Für $p_{a l p_{1}}$ und $p_{a l v_{2}}$ in Millimeter $\mathrm{Hg}$ und $\mathrm{C}$ in Litern erhält man annähernd $k_{e l}$ in Zentimeter Wassersäule.

Die so bestimmten $k_{e l}$-Werte sind für Männer vom zweiten bis Anfang des fünften Dezenniums wenig verschieden. Neun Messungen liegen zwischen 11,9 und 14,7 . 
In höherem Alter sinkt $k_{e l}$, um beim Altersemphysem noch etwa die Hälfte des früheren Wertes zu betragen. (Durchschnitt für 18 Emphysematiker: $k_{\ell l}=7,1$ ).

6. Die elastische Spannung in der Brusthöhlenumgebung $\left(p_{\epsilon l}\right.$ thor $)$ ist aus den $\Sigma_{p_{\ell l}}$-Werten zu berechnen, durch Subtraktion der für die einzelnen Dehnuıgszustände annähernd bekannten Retraktionskraft (Donders, Cloetta) der Lungen.

Aus den $\Sigma p_{c l}$-Kurven im Sitzen, Liegen und bei Anbringung einer elastischen Binde ergeben sich die entsprechenden $p_{t l}$ thorKurven, die tiefer und etwas flacher liegen als die ersteren. (Fig. 2.)

Bei der Muskelerschlaffung ist $p_{\epsilon l}$ thor an allen Oberflächeneinheiten der Brusthöhle gleich gross. Bei Muskelwirkung ist der Durchschnittswert von $p_{\epsilon l}$ thor wenig verschieden von dem bei Muskelerschlaffung.

Für die Wahl des Atemtypus ist massgebend die Grösse der

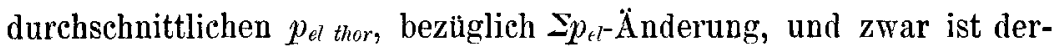
jenige Typus herrschend, der unter den gegebenen Verhältnissen inspiratorisch die geringste Spannungszunahme bedingt.

7. Die maximale inspiratorische und exspiratorische Kraft ist abhängig vom Dehnungszustand der Atemorgane (Fig. 3; Kurve $I$ und $I I)$.

Die maximale muskuläre Kraft ist aus den letzteren Werten durch Subtraktion der elastischen Kraft $\left(\Sigma_{p_{\epsilon}}\right)$ zu berechnen. Ihre Abhängigkeit vom Dehnungszustand (Fig. 3; Kurve III und IV) ist durch die ändernde Distanz von Ursprung und Ansatz der Atemmuskeln und durch die ändernde Grösse der Brusthöhlenoberfläche zu erklären.

8. Die an der gesamten Brusthöhlenoberfläche wirkenden Kräfte berechnen sich aus den Kräften an der Flächeneinheit und der Oberflächengrösse $(O)$. Bei einem gegebenen Dehnungszustand ist unter statischen Verhältnissen: $O \cdot p_{\text {mutsk }}=O \cdot \Sigma_{p_{e l}}+O \cdot p_{a l l}$.

Die Grösse der einzelnen Werte bei den verschiedenen Dehnungszuständen ist für die Versuchsperson durch die Kurven von Fig. 4 dargestellt. Die maximale Kraftentfaltung beträgt für die Versuchsperson inspiratorisch $220 \mathrm{~kg}$, exspiratorisch $305 \mathrm{~kg}$. 